\title{
Prostate-targeted radiosensitization via aptamer-shRNA chimeras in human tumor xenografts
}

\author{
Xiaohua Ni, ${ }^{1}$ Yonggang Zhang, ${ }^{2}$ Judit Ribas, ${ }^{1}$ Wasim H. Chowdhury, ${ }^{1}$ Mark Castanares, ${ }^{1}$ \\ Zhewei Zhang, ${ }^{2}$ Marikki Laiho,2 Theodore L. DeWeese,1,2,3 and Shawn E. Lupold1,2,3 \\ 1James Buchanan Brady Urological Institute, 2Department of Radiation Oncology and Molecular Radiation Sciences, and \\ ${ }^{3}$ Sidney Kimmel Comprehensive Cancer Center, Johns Hopkins University School of Medicine, Baltimore, Maryland, USA.
}

\begin{abstract}
Dose-escalated radiation therapy for localized prostate cancer (PCa) has a clear therapeutic benefit; however, escalated doses may also increase injury to noncancerous tissues. Radiosensitizing agents can improve ionizing radiation (IR) potency, but without targeted delivery, these agents will also sensitize surrounding normal tissues. Here we describe the development of prostate-targeted RNAi agents that selectively sensitized prostate-specific membrane antigen-positive (PSMA-positive) cells to IR. siRNA library screens identified DNA-activated protein kinase, catalytic polypeptide $(D N A P K)$ as an ideal radiosensitization target. DNAPK shRNAs, delivered by PSMA-targeting RNA aptamers, selectively reduced DNAPK in PCa cells, xenografts, and human prostate tissues. Aptamer-targeted DNAPK shRNAs, combined with IR, dramatically and specifically enhanced PSMA-positive tumor response to IR. These findings support aptamer-shRNA chimeras as selective sensitizing agents for the improved treatment of high-risk localized PCa.
\end{abstract}

\section{Introduction}

An estimated 1 in 6 men will be diagnosed with prostate cancer (PCa). Although the majority of these men can be successfully treated with surgery or radiation therapy, approximately $20 \%-40 \%$ will biochemically recur within 10 years of treatment (1). This risk of recurrence is elevated to approximately $50 \%$ for men with locally advanced disease, a condition that is primarily managed by radiation therapy $(2,3)$. Thus, new technologies that improve the therapeutic index of radiation therapy for local disease have the opportunity to significantly affect the morbidity and mortality of PCa.

Ionizing radiation (IR) causes multiple types of cellular injury, of which DNA double-strand breaks (DSBs) are considered the most cytotoxic (4). Naturally occurring mutations in genes that sense or repair DNA damage are associated with increased sensitivity to IR $(5,6)$. Chemical or siRNA inhibition of DNA repair proteins, such as ataxia telangiectasia mutated (ATM) or NBS1, also results in cellular hypersensitivity to IR (7-9). Although these approaches have potential, they lack a means to selectively target cancer cells or specific tissues. Prostate-targeted radiosensitization approaches have the potential to both increase the therapeutic effect of IR and reduce radiation-associated damage to other pelvic tissues.

RNAi is a promising new therapeutic approach. The challenge for translating RNAi therapy is delivery, particularly for specific cell types. One developing delivery approach is RNA aptamers, which are nuclease-stabilized targeting molecules capable of binding ligands in much the same way as antibodies (10-12). We have previously developed prostate-specific membrane antigen-targeted (PSMA-targeted) RNA aptamers (13), which are capable of targeting drugs, nanoparticles, and toxins to PSMA-expressing PCa cells and tumors (14-18). When conjugated to siRNAs and shRNAs, these PSMA aptamers are also capable of delivering cell-selective gene knockdown (19-23). Because PSMA is highly expressed in

Conflict of interest: The authors have declared that no conflict of interest exists. Citation for this article: JClin Invest. 2011;121(6):2383-2390. doi:10.1172/JCI45109. nearly all localized prostate tumors $(24,25)$, we hypothesized that PSMA-targeted aptamer-shRNA chimeras could be used to inhibit DNA repair pathways in prostatic cells for enhanced radiation therapy of locally advanced PCa.

\section{Results}

High-throughput siRNA screen of DNA repair pathway genes for radiosensitization. To explore the combination of IR with siRNA, we screened a custom siRNA library against 249 mRNAs, primarily encoding critical DNA repair proteins. The goal was to identify radiosensitizing target genes and corresponding siRNAs. Radiosensitization was calculated as percent increased cell death associated with a gene-specific siRNA after radiation therapy (6 Gy) compared with irradiated cells transfected with a control siRNA. Candidate targets were defined as those for which 2 separate siRNAs, targeting the same gene, enhanced radiation-induced cell death above the SD of the library mean (Figure 1 and Supplemental Table 1; supplemental material available online with this article; doi:10.1172/JCI45109DS1). In total, 10 candidate genes were identified as PCa radiosensitization targets, 6 of which were separately confirmed by clonogenic survival assays (Figure 2). Effective target gene knockdown at the time of irradiation was confirmed by quantitative RT-PCR (qRT-PCR; Supplemental Figure 1). The dose-modifying factor $\left(\mathrm{DMF}_{0.1}\right)$ was calculated for each target as the ratio of IR dose required for $90 \%$ cell kill by control siRNA versus gene-specific siRNA, and 3 target genes - specifically, the catalytic subunit of DNA-activated protein kinase, catalytic polypeptide (DNAPK); mitotic spindle assembly checkpoint protein MAD2B (MAD2L2); and breast cancer type 2 susceptibility protein $(B R C A 2)$ - achieved $D \mathrm{MF}_{0.1}$ of 1.6 by siRNA inhibition. The remaining 3 target genes achieved $\mathrm{DMF}_{0.1}$ of 1.3 in clonogenic survival assays. All 6 candidate genes were verified as radiosensitizing targets in a second cell line, the PSMA-positive cell and tumor model LNCaP (Supplemental Figure 2). Interestingly, the siRNA screen primarily identified genes involved in DSB repair, except $R A D 23 B$, a gene involved in excision repair (26). 


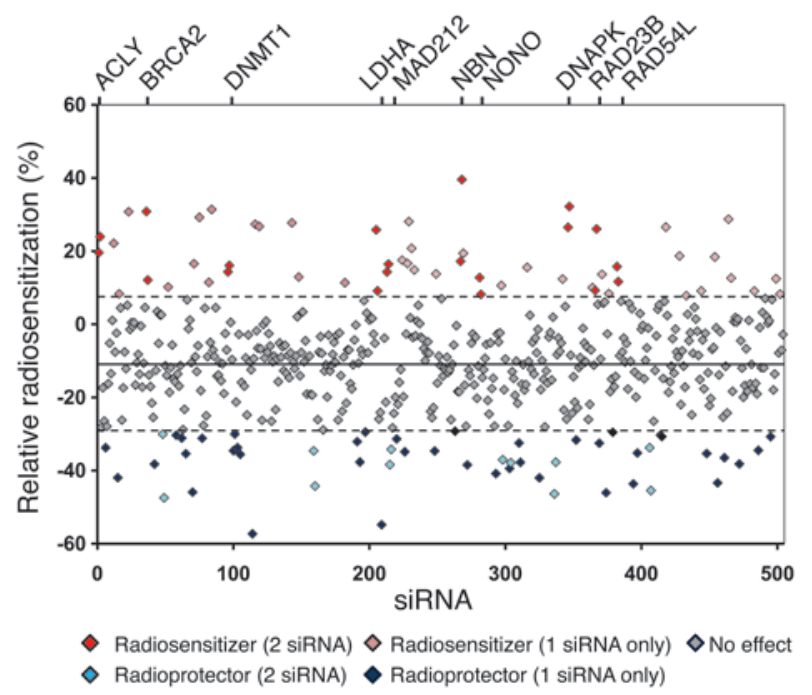

Aptamer-shRNA chimera selective targeting. Candidate and previously identified radiosensitizing siRNAs (8) were then linked to the PSMA-targeting A10-3 aptamer for selective cell delivery. We designed aptamer-shRNA chimeras as a single intact nuclease-stabilized 2' fluoro-modified pyrimidine transcript. The 3 '-terminus of the A10-3 aptamer was conjugated to the passenger (sense) strand of the siRNA, followed by a 10-mer loop sequence and then by the guide or silencing (antisense) strand of the siRNA. The secondary structures of each aptamer-shRNA chimera were evaluated by mFold (http://mfold.rna.albany.edu/) to predict proper folding of the aptamer portion (Figure 3A). Control chimeras were generated with nonspecific shRNAs (referred to herein as A10-3Con) or nontargeting aptamer portions (denoted by the prefix Neg-followed by the target gene; Figure $3 \mathrm{~B}$ ). LNCaP cells were

\section{Figure 1}

Identification of radiosensitization target genes and siRNAs. siRNA library screen for candidates in DU145 cells. Cells were transfected with library siRNAs or controls. Transfected cells were untreated or irradiated (6 Gy) 72 hours later. Radiosensitization was calculated as percent increased cell death associated with a gene-specific siRNA after radiation therapy compared with irradiated cells transfected with a control siRNA. Each diamond represents an siRNA (triplicate, average) organized alphabetically. Mean library radiosensitization and SD are shown by solid and dashed lines, respectively.

treated with aptamer-shRNA chimeras, in the absence of transfection reagents, and changes in target gene mRNA were evaluated by qRT-PCR relative to untreated cells. The efficiency of chimera A10-3-DNAPK was first evaluated at 4, 40, and $400 \mathrm{nM}$ in LNCaP cells, and $400 \mathrm{nM}$ was determined to be the most effective dose (Figure 3C). Within 48 hours of treatment at $400 \mathrm{nM}$, aptamertargeted shRNA caused significant reductions in DNAPK, BRCA2, and ATM mRNA levels (Figure 3, D-F). A10-3-Con and aptamer control chimeras (Neg-DNAPK, Neg-BRCA2, and Neg-ATM) had no detectable effect on target mRNA levels. Transfected siRNA served as a positive control for knockdown of each gene. Since delivered shRNAs could potentially induce nonspecific inflammatory responses that cause cellular toxicity (27), we then evaluated by ELISA whether INF- $\beta$ was induced in LNCaP cells either transfected with DNAPK siRNAs or treated with aptamer-shRNA chimeras. The applied treatment conditions caused no detectable increases in INF- $\beta$ (Supplemental Figure 3).

In vitro and in vivo PSMA selectivity and aptamer-shRNA chimera processing. To further confirm PSMA-selective targeting, a second and previously described isogenic cell model of PSMA-expressing PC3 cells (PC3-PIP) and PSMA-negative control cells (PC3-Flu) (28) was subjected to aptamer-shRNA chimera treatment. A10-3-DNAPK treatment selectively reduced DNAPK levels in PC3-PIP cells, but
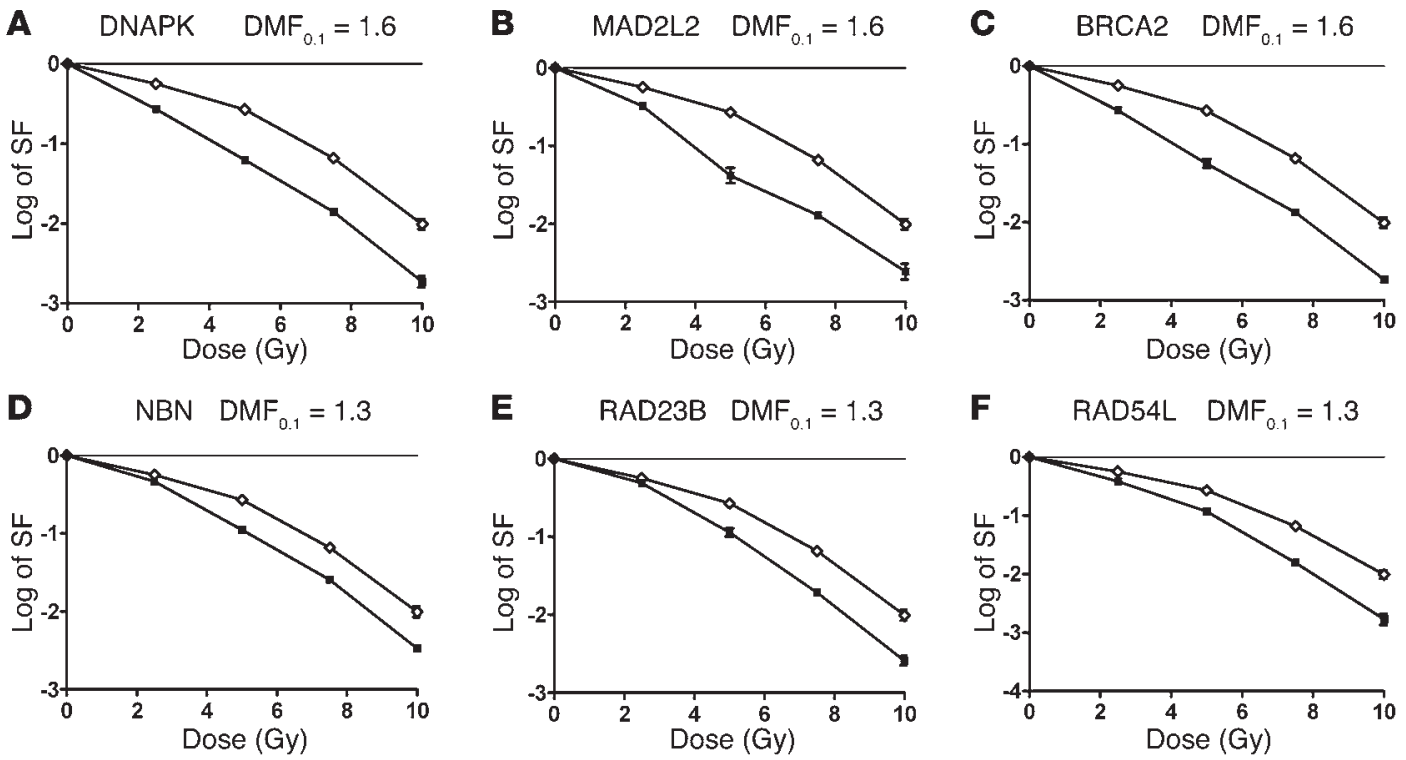

Figure 2

Clonogenic survival assays. (A-F) Radiosensitization was confirmed by clonogenic survival assays $(n=3)$. DU145 cells were reverse transfected with $5 \mathrm{nM}$ control siRNA or (A) DNAPK, (B) MAD2L2, (C) BRCA2, (D) NBN, (E) RAD23B, or (F) RAD54L siRNA 72 hours prior to irradiation. $\mathrm{DMF}_{0.1}$ values are shown for each. Open diamonds, control siRNA; closed squares, candidate siRNA. SF, surviving fraction. 
A

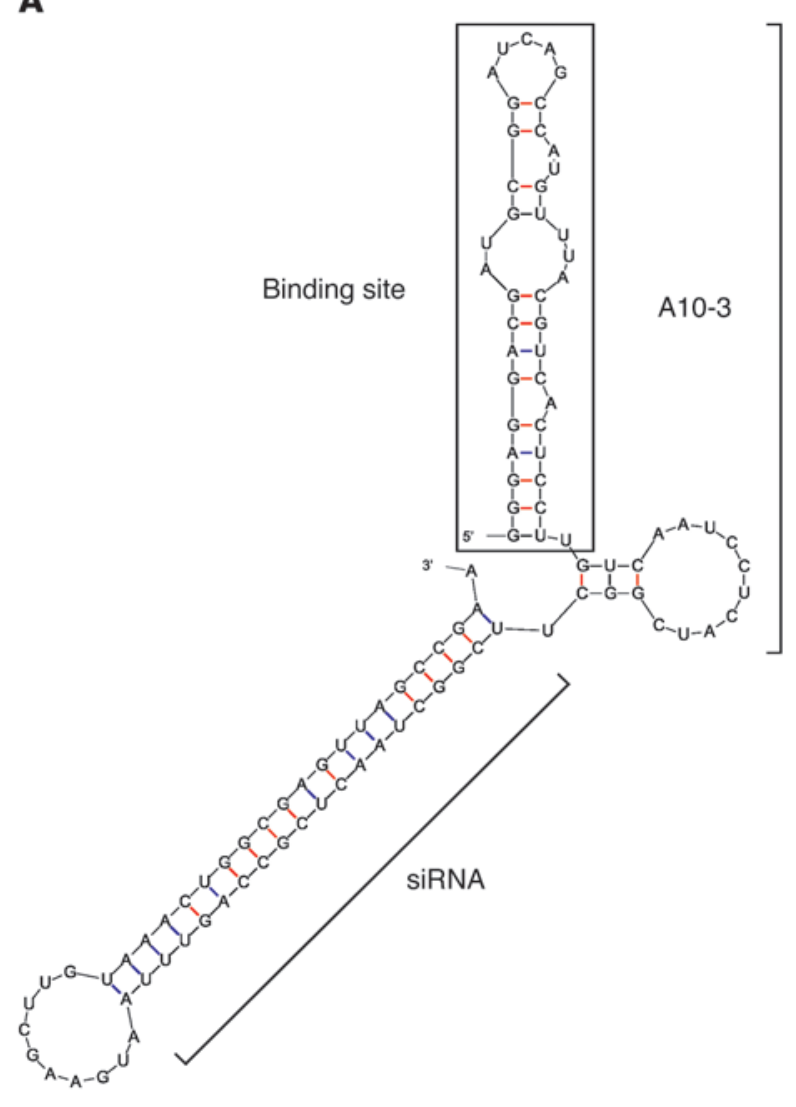

B

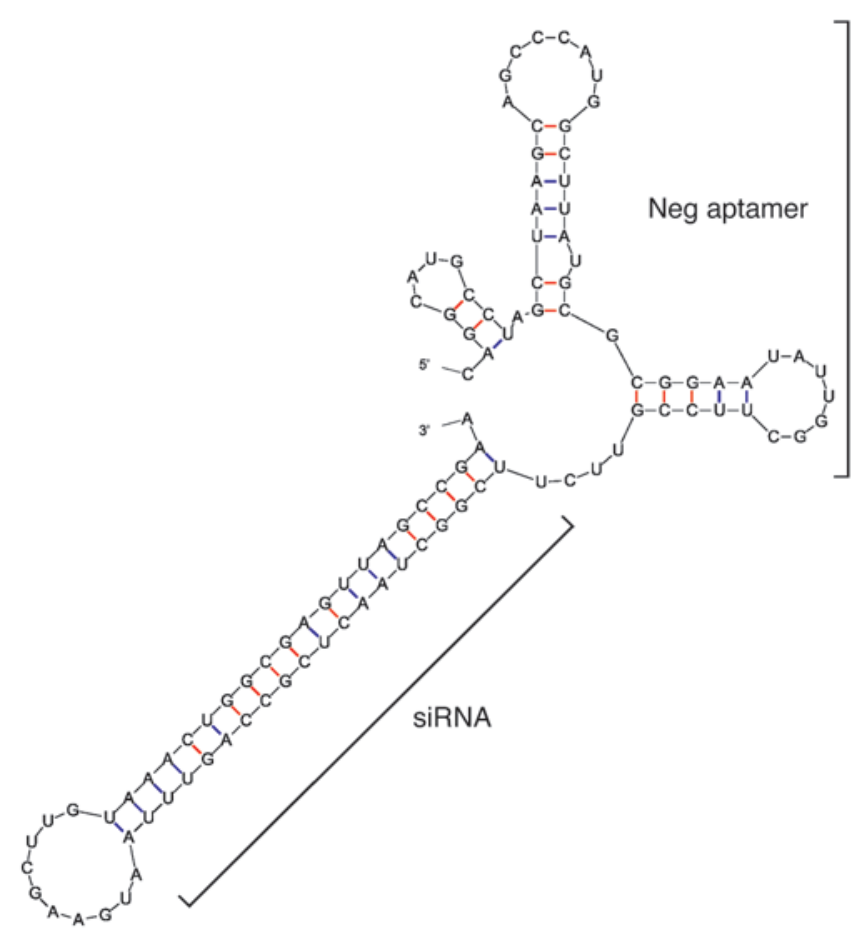

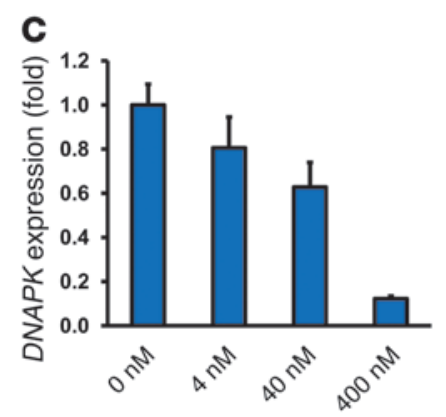

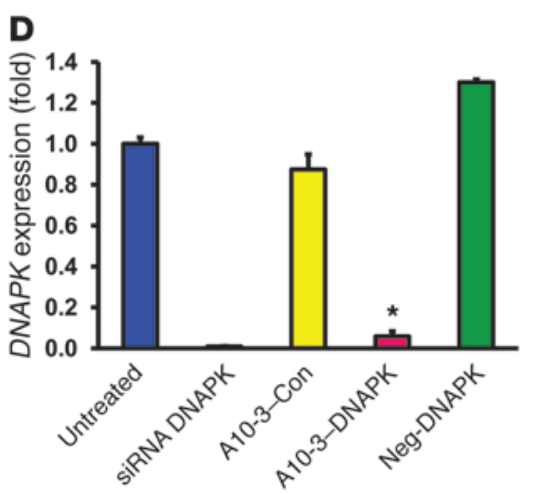

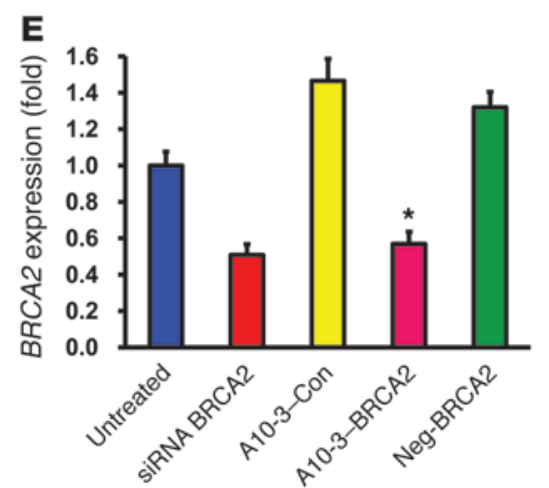

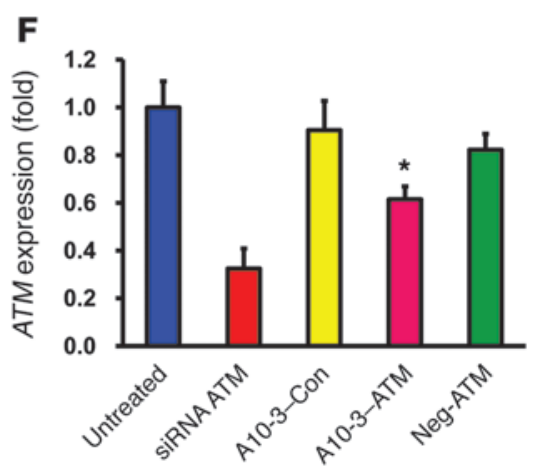

Figure 3

Aptamer-shRNA chimeras and PSMA-targeted DNA repair gene knockdown. (A and B) Secondary structure of (A) A10-3-DNAPK and (B) Neg-DNAPK. Aptamer and shRNA portions are shown by brackets. (C-F) Aptamer-shRNA chimera-mediated RNAi in the absence of transfection reagents. (C) LNCaP cells were treated with 0, 4, 40, or $400 \mathrm{nM} \mathrm{A10-3-DNAPK} \mathrm{for} 48$ hours, and DNAPK mRNA levels were quantified by qRT-PCR. LNCaP cells were treated with $400 \mathrm{nM}$ of (D) A10-3-DNAPK, (E) A10-3-BRCA2, or (F) A10-3-ATM for 48 hours. Respective target gene mRNA levels were quantified by qRT-PCR. Appropriate siRNAs were transfected $(100 \mathrm{nM})$ as positive controls. Expression is normalized to GAPDH. Mean \pm SEM $(n=3) .{ }^{*} P<0.05$. 

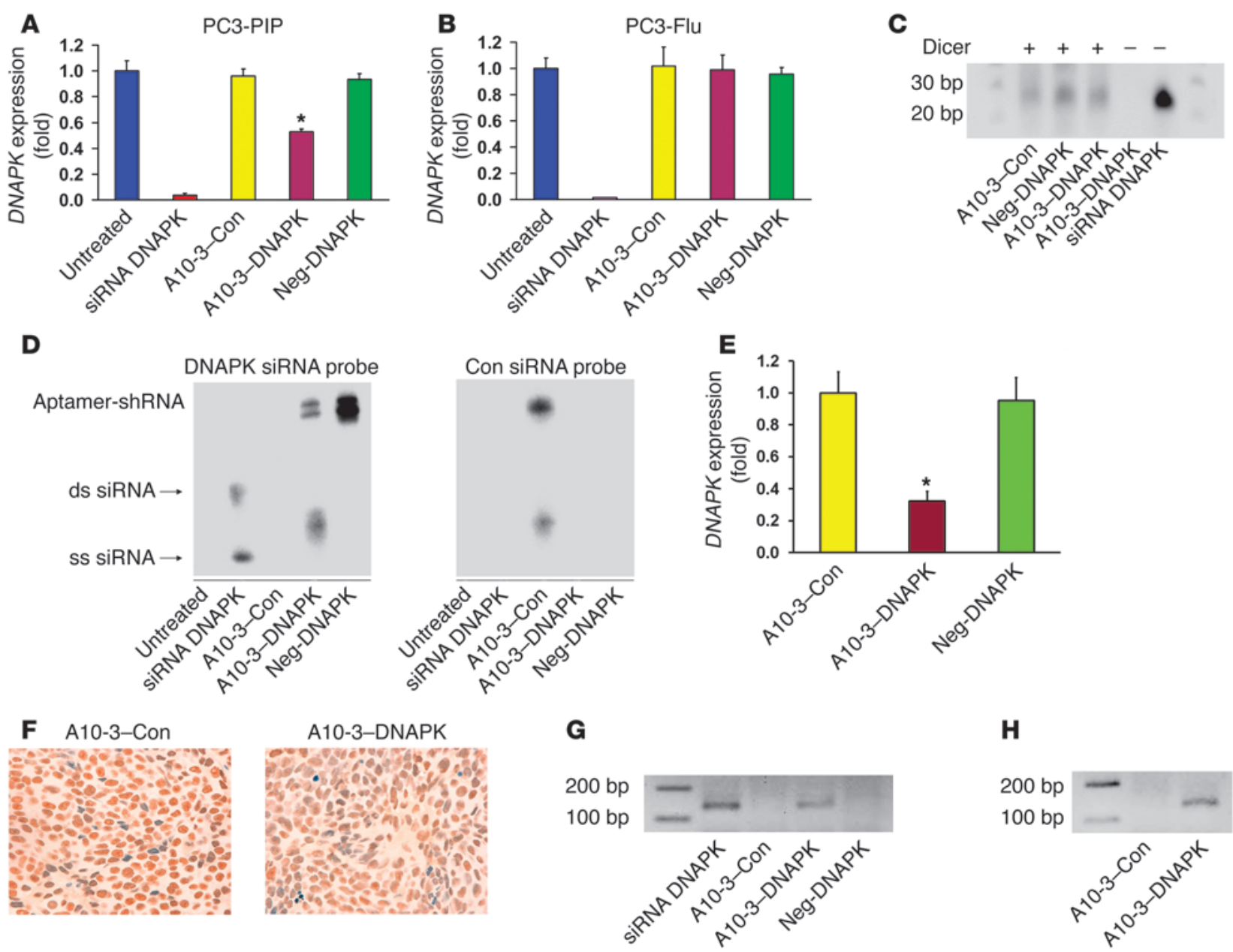

\section{Figure 4}

PSMA selectivity and aptamer-shRNA chimera processing. (A and B) PSMA selectivity. (A) PC3-PIP or (B) PC3-Flu cells were treated with $400 \mathrm{nM}$ aptamer-shRNA chimeras for 48 hours, and DNAPK expression was quantified by qRT-PCR. siRNA DNAPK (100 nM transfected) was included as a positive control. Expression is normalized to GAPDH. Mean \pm SEM $(n=3)$. ${ }^{*}<0.05$. (C) Aptamer-shRNA chimera processing by Dicer in vitro. Cleavage products were analyzed by denaturing PAGE and ethidium bromide staining. Image is inverted for clarity. (D) Cellbased RNAi processing assay. LNCaP cells were treated with $400 \mathrm{nM}$ aptamer-shRNA chimeras, and RNA was extracted 48 hours later for Northern blot assay. Probes are specific to corresponding antisense siRNAs. ds, double-stranded; ss, single-stranded. (E and F) Targeted in vivo knockdown. Subcutaneous LNCaP tumors were injected with aptamer-shRNA chimeras (200 pmol/injection) on days -3 and -2 and harvested on day 0 , and DNAPK expression was determined. (E) qRT-PCR. Mean \pm SEM. ${ }^{*} P<0.05$. (F) Immunohistochemistry. Original magnification, $\times 400$. (G and $\mathbf{H}) 5^{\prime}$-RACE PCR analysis to assess siRNA-mediated cleavage of DNAPK. (G) LNCaP cells transfected with DNAPK siRNA or with A10-3-DNAPK chimeras produced a specific DNAPK cleavage product. $(\mathbf{H})$ In vivo treatment of LNCaP xenografts with A10-3-DNAPK chimera resulted in siRNA-mediated DNAPK cleavage.

not in PC3-Flu cells (Figure 4, A and B). We next evaluated the processing of aptamer-shRNA chimeras by RNAi machinery. Aptamer-shRNA chimeras were incubated in the presence or absence of recombinant human Dicer for 1 hour at $37^{\circ} \mathrm{C}$. shRNA cleavage products were obtained in samples treated with Dicer, whereas no cleavage products were detected in its absence (Figure 4C). We further interrogated aptamer-shRNA chimera processing in an intact cell model. LNCaP cells were treated with A10-3 aptamer-shRNA chimeras in the absence of transfection reagents and evaluated for the presence of the desired siRNA product by Northern blotting. A10-3-DNAPK and A10-3-Con were effectively internalized and processed to produce antisense siRNAs (Figure 4D). Collectively, these results support that A10-3 aptamer-shRNA bound PSMA, were internalized into cells, and were processed by RNAi machin- ery, whereas Neg-DNAPK could not enter cells to be processed. In the absence of extensive cell washing, the unprocessed chimeras were detectable by Northern blot, and levels indicated that approximately half of the A10-3 aptamer-shRNA chimeras were internalized and processed to the mature siRNAs in the experimental time period. Processed siRNAs from aptamer-shRNA chimeras resulted in products that were slightly different compared with reference siRNA. Next, aptamer-targeted DNAPK RNAi was evaluated in vivo. LNCaP xenografts were established subcutaneously in nude male mice and treated with $200 \mathrm{pmol}$ of targeted and control aptamer-shRNA chimeras by 2 consecutive intratumoral injections. qRT-PCR and immunohistochemistry demonstrated reduction of DNAPK mRNA and DNAPK protein after treatment with A10-3DNAPK, but not controls (Figure 4, E and F). Quantification of 
A
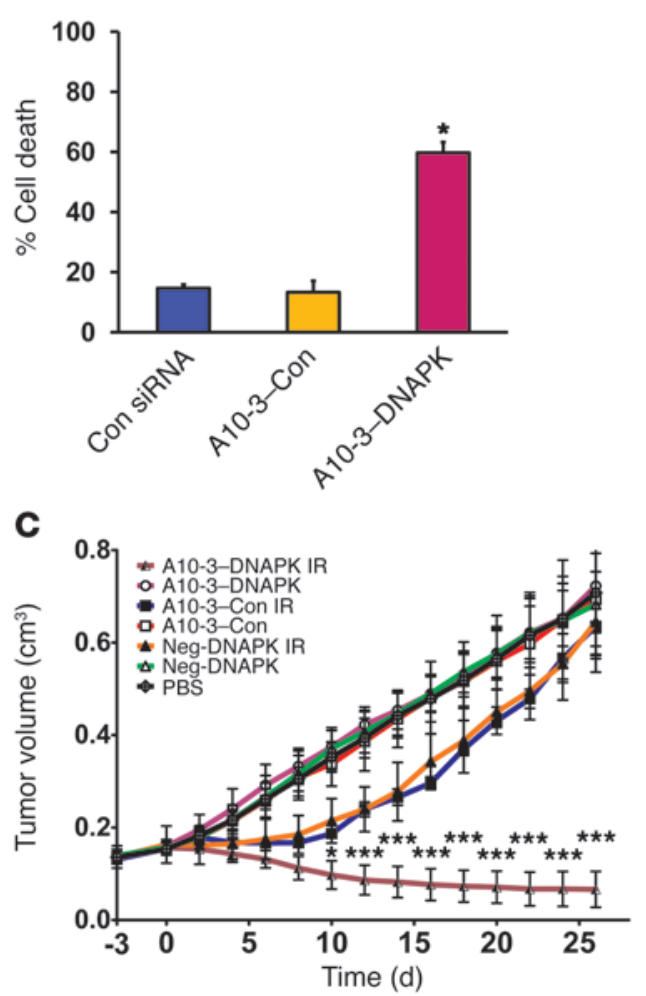

B

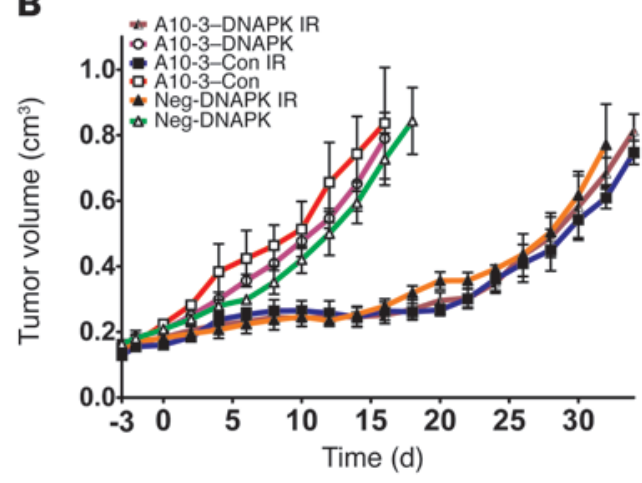

D

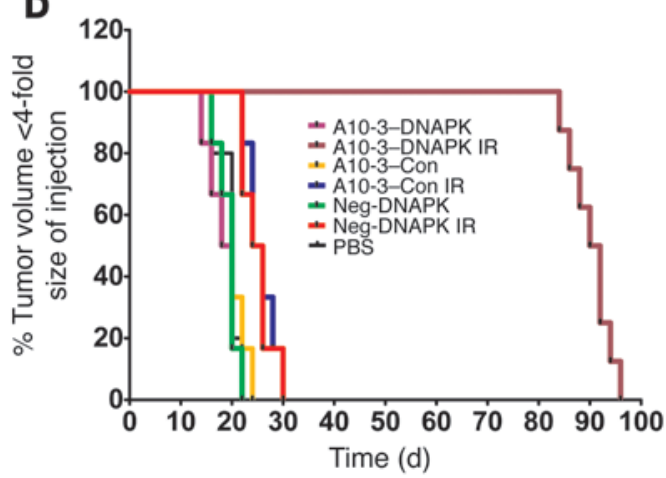

Figure 5

Radiosensitization in PCa cell and tumor models. (A) In vitro radiosensitization. LNCaP cells treated with 400 nM A10-3-DNAPK or A10-3-Con or transfected with control siRNA were irradiated 48 hours later with 6 Gy IR, and cell viability was assessed 12 days later by MTS. Percent cell death is relative to nonirradiated cells. (B-D) In vivo radiosensitization. Established tumors were treated with aptamer-shRNA chimeras (days -3 and -2) and either 6 Gy IR or no radiation (day 0). (B) PC3 tumor model ( $n=3$ per group). A10-3-DNAPK provided no significant therapeutic benefit to nonirradiated or irradiated PC3 tumors. Radiation similarly affected growth in all treatment groups. Mean \pm SEM. (C) LNCaP tumor model ( $n \geq 6$ per group). Radiation similarly affected growth in all treatment groups except irradiated A10-3-DNAPK. ${ }^{*} P<0.05,{ }^{* * *} P<0.001$, A10-3DNAPK IR vs. A10-3-Con IR and A10-3-DNAPK IR vs. Neg-DNAPK IR; 2-way ANOVA. Mean \pm SEM. (D) Extension of tumor quadrupling for LNCaP tumor model. Events (animals whose tumor volume was not yet 4-fold the size at injection) were plotted by Kaplan-Meier curve. $P<0.01$, A10-3-Con IR vs. A10-3-Con and Neg-DNAPK IR vs. Neg-DNAPK; $P<0.0001$, A10-3-DNAPK IR vs. A10-3-Con IR and A10-3-DNAPK IR vs. Neg-DNAPK IR; log-rank (Mantel-Cox) test.

total DNAPK protein staining per nuclear area by FrIDA image analysis (29) indicated a $52 \%$ protein reduction in A10-3-DNAPKtreated tumors compared with A10-3-Con treatment. Finally, we confirmed that aptamer-shRNA chimera-mediated DNAPK knockdown occurs through RNAi by identifying DNAPK mRNA cleavage products with $5^{\prime}$-rapid amplification of cDNA ends (5'-RACE) in cell line and animal model treatments (Figure 4, G and H). These studies establish aptamer and PSMA-selective targeted RNAi of DNA repair protein production in prostate tumor models.

Aptamer-targeted radiosensitization in buman PCa cell and tumor models. The most potent aptamer-shRNA chimera targeted the catalytic subunit of DNAPK. This chimera was therefore selected for targeted radiosensitization studies in LNCaP cells. LNCaP, rather than PC3-PIP, was selected for these studies due to the cells' consistent and high-level PSMA expression (Supplemental Figure 4). Treatment of LNCaP cells with A10-3-DNAPK in the absence of transfection reagents significantly increased cell death after IR compared with controls (Figure 5A). These results support the hypothesis of aptamer-targeted radiosensi- tization. LNCaP tumors and PC3 tumors were then established subcutaneously in male nude mice and intratumorally injected twice with 200 pmol targeted or control aptamer-shRNA chimeras (days -3 and -2 ). Half of each cohort then received either no radiation treatment or a single radiation treatment ( $6 \mathrm{~Gy}) 2$ days after aptamer-shRNA chimera injection (day 0$)$. No differences in tumor volume were observed between nonirradiated cohorts (Figure 5, B and C; see Supplemental Figure 5 for individual tumor curves), which suggests that chimera treatment alone had no detectable therapeutic effect in either tumor model. In both LNCaP and PC3 tumor models, irradiated tumors treated with control aptamer-shRNA chimeras resulted in a significant but temporary reduction in tumor volume compared with nonirradiated samples (Figure 5D). Notably, the combination of A10-3DNAPK and radiation resulted in a significant and extended tumor response in LNCaP tumors, but not PC3 tumors. This combination treatment dramatically extended the time to reach quadruple tumor volume, by approximately 10 weeks compared with 1 week in tumors treated with radiation and control aptamer- 

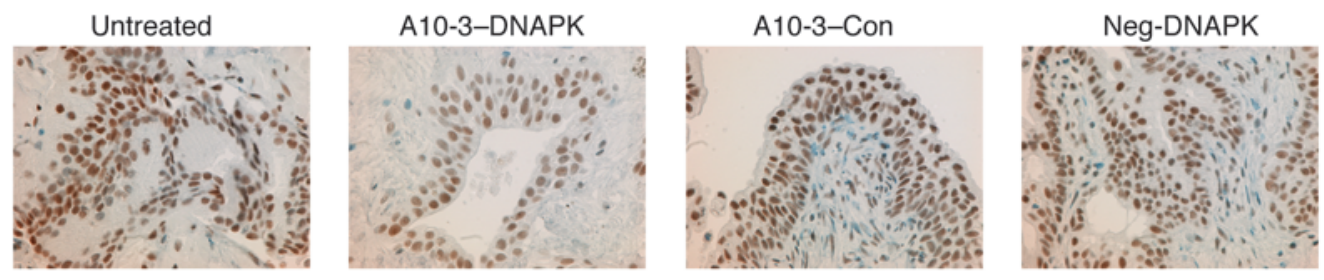

\section{Figure 6}

Ex vivo treatment of human prostate tissue. Sections of normal human prostate tissue were obtained from fresh radical prostatectomy specimens and maintained ex vivo. These were treated with $200 \mathrm{nM}$ aptamer-shRNA chimeras, and DNAPK levels were detected by immunohistochemistry 48 hours after treatment. Quantitative image analysis determined a 25\% reduction in DNAPK staining for A10-3-DNAPK-treated samples. Original magnification, $\times 400$.

shRNA chimeras (Figure 5D). Thus, in cell and tumor models, aptamer-targeted knockdown of DNAPK selectively enhanced radiosensitivity and increased therapeutic effect.

Aptamer-shRNA chimera-specific knockdown of the target gene in buman prostate tissue. Finally, to determine whether aptamer-shRNA chimeras would be effective in human tissue, we used a unique human tissue model in which fresh sections of histologically normal human prostate were obtained from radical prostatectomy specimens and immediately maintained ex vivo (30). PSMA expression in these noncancerous tissue sections were confirmed by qRT-PCR prior to treatment. Tissue was then treated with A10-3DNAPK and control aptamer-shRNA chimeras in the absence of transfection reagents. Quantitative image analysis found DNAPK immunostaining to be decreased by $25 \%$ in normal prostate epithelial cells 2 days after treatment with A10-3-DNAPK compared with those treated with control aptamer-shRNA chimeras (Figure 6). Thus, aptamer-shRNA chimeras are effective in treating human prostate tissues. We anticipate that knockdown in cancer specimens would be much more substantial because of known elevated PSMA expression in primary prostate tumors.

\section{Discussion}

Here we describe a model to test the hypothesis that the therapeutic index for local treatment of PCa can be improved by selectively sensitizing PCa cells to IR. The therapeutic strategy to deliver doseescalated radiation therapy to the prostate, historically considered as more than approximately $70 \mathrm{~Gy}$, has been constrained by the limited tolerance of the urinary tract and rectum $(31,32)$.Thus, strategies that provide the benefits of dose-escalated radiation without the associated risks to normal tissue, the concomitant expensive hightech infrastructure, and/or the added use of androgen suppression could have a significant impact on PCa morbidity and mortality.

DNA repair pathways are an attractive therapeutic target for radiosensitization. DSBs are generally regarded as the most lethal of all DNA lesions; if unrepaired, they severely threaten not only the integrity of the genome, but also the survival of the organism (33-35). To combat the intricate nature of DSBs, complex repair pathways have evolved. Thus, multiple steps of DSB repair pathways, enzymes, and proteins are potential targets for RNAiinduced radiosensitization therapy. Here we report what may be the first high-throughput screen of DNA repair pathways by siRNA in combination with radiation therapy. Of 249 mRNAs screened, only 6 candidates were identified by at least 2 siRNAs and confirmed in separate PCa cell line models. These target genes are likely good candidates for radiosensitization in other tissue and cancer types.
Since the discovery of RNAi, this pathway has been widely recognized as a new frontier for human therapeutics, and many human clinical trials using this technology are currently planned or in progress. As with other therapeutic approaches, there is a need for selective tissue targeting to minimize damage to normal tissues (36-39). We have previously developed PSMA-targeting aptamers as a means to selectively deliver therapeutic and imaging agent to PCa cells (13). Several groups have independently used these aptamers to target therapeutics, including siRNAs and shRNAs (19-21, 23). Here, we have also extended on the initial work by generating 2 ' fluoro-modified pyrimidine aptamer-shRNA chimeras as selective radiosensitizing agents. The conjugates retained PSMA targeting ability, and the shRNA portion of the aptamershRNA chimera was effectively processed by RNAi machinery to the predicted antisense siRNA. There was a slight difference in the siRNA product size compared with the reference siRNA, which may be caused by 2 '-fluoro-modifications or by cleavage somewhere in the aptamer loop. Similar size differences have been seen in aptamer-siRNA chimera studies (20). The resulting siRNA product was then free to degrade the target transcript at the predicted site, as demonstrated by 5 -RACE. These results support that aptamer-shRNA chimeras can be developed for virtually any target gene, including those that sensitize cancer cells to standard therapeutic approaches. Some advantages of aptamer-shRNA chimeras are their simplicity, potential for chemical synthesis, safety, and low toxicity (40-42).

The goal of this study was to develop aptamer-shRNA chimeras as tools that may benefit men being treated with radiation therapy for localized PCa. Although radiation therapy of low-risk PCa is highly successful, there are treatment-related risks that could be diminished with a radiation dose-reducing strategy predicated on our aptamer-shRNA chimera approach. Moreover, treatment efficacy of higher-risk local tumors could be improved with radiosensitization while also minimizing side effects. Preliminary studies here suggested that $D N A P K$ knockdown could improve therapeutic efficacy by almost 10 -fold (Figure $2 \mathrm{~A}$ ). There is further potential to improve this technology. Dassie and colleagues recently reported a truncated and more potent form of PSMA aptamer-siRNA chimera that was effective at knocking-down polo-like kinase 1 ( $p l k 1$ ) with low nanomolar concentrations (20). This smaller chimera was capable of knocking down plk1 in established subcutaneous tumors after repeated intraperitoneal injection. Thus, there is potential that the current A10-3-DNAPK chimeras could be improved by modification and be applicable to metastatic disease. Inhibition of DNA repair pathways can also sensitize cells to chemotherapeutics, such as alkylating agents and topoisomerase inhibitors, therefore 
providing a potential mechanism for systemic chemosensitization (8). We are therefore looking to develop next-generation aptamer chimeras that may benefit men with metastatic PCa.

In summary, our results indicate that the aptamer-shRNA chimeras retain PSMA-selective targeting, proper Dicer shRNA processing, and subsequent target gene knockdown in PCa cells, tumor xenografts, and normal human prostatic tissue models. This targeted treatment markedly enhanced the benefits of radiation therapy in both cellular and tumor models, supporting the concept that aptamer-shRNA chimeras may be exploited as a means to improve radiation therapy for locally advanced PCa.

\section{Methods}

Cell culture. PCa cell lines DU145 (ATCC no. HTB-81), LNCaP (ATCC no. CRL1740), PC3 (ATCC no. CRL-1435), PC3-PIP, and PC3-Flu (gift of W. Heston, Lerner Research Institute, Cleveland, Ohio, USA) were grown in RPMI 1640 supplemented with $10 \% \mathrm{FBS}$ and maintained at $37^{\circ} \mathrm{C}$ and $5 \% \mathrm{CO}_{2}$.

$D N A$ repair siRNA library screen. A custom siRNA library included 496 siRNAs targeting 249 genes and controls (Qiagen). $2 \times 10^{3}$ DU145 were Hiperfect reverse transfected (Qiagen) in triplicate in 96-well plates formatted with $5 \mathrm{nM}$ siRNA. 72 hours later, cells were irradiated (6 Gy in a Gammacell 40 [Nordion] $137 \mathrm{Cs}$ radiator at approximately $0.6 \mathrm{~Gy} / \mathrm{min}$ ) and grown for 72 hours. Cell viability was quantified by 3-(4,5-dimethylthiazol-2-yl)-5-(3-carboxymethoxyphenyl)-2-(4-sulfophenyl)-2H-tetrazolium (MTS; Promega). Irradiated and nonirradiated viability was normalized to the control siRNA for each siRNA, and radiosensitization was determined as the ratio of increased cell death relative to the control siRNA. Significance was assigned as $P<0.05$ by Student's $t$ test. Candidate sensitizing siRNAs were confirmed by repeat assays and clonogenic survival assays.

Clonogenic survival assays. Clonogenic survival assays were confirmed in a larger format, in which $1.7 \times 10^{5}$ DU145 cells were reverse transfected with $5 \mathrm{nM}$ control and candidate siRNAs and grown for 72 hours, after which cell dilutions were plated into $100-\mathrm{mm}$ culture dishes and irradiated immediately. Exposures were carried out as described above. The cells were grown for 14 days and stained with crystal violet; colonies with greater than 30 cells were scored, and survival fraction was calculated.

qRT-PCR. mRNA $(1 \mu \mathrm{g})$ from PCa cells treated with the various siRNAs or aptamer-shRNA chimeras was reverse transcribed using QuantiTect Reverse Transcription Kit (Qiagen). Sybr green-based real-time qRT-PCR was performed using SYBR GreenER qPCR SuperMix (Invitrogen) according to the manufacturer's instructions. All reactions were done in triplicate. Standard curves were generated by serial dilution of each sample, and the relative amount of target gene mRNA was normalized to GAPDH mRNA (see Supplemental Table 2 for primers).

Aptamer-shRNA chimeras. Aptamer-shRNA chimeras were generated as follows. PSMA-targeting (A10-3) or nontargeting (Neg) template primers (Supplemental Table 3) were fused to a corresponding shRNA by PCR with Pfu polymerase (NEB). These first DNA templates were column purified (Qiagen) and separately used as templates for secondary PCR with A10-3 or Neg 5'-primer and the appropriate second primer for each gene by Taq polymerase (Qiagen). After column purification, products were TA cloned (Promega) and sequenced. PCR products from plasmid or the secondary PCR were used as templates for DuraScribe T7 transcription according to the manufacturer's instructions (Epicenter Biotechnologies). Aptamer-shRNA chimeras (Supplemental Table 4) were purified by gel electrophoresis (13).

Aptamer-shRNA chimera-mediated gene silencing and radiosensitization assay. $2 \times 10^{5}$ cells (LNCaP, PC3-PIP, or PC3-Flu) were Hiperfect transfected with $100 \mathrm{nM}$ siRNA (Supplemental Table 5) in 6-well plates or treated with 4,40 , or $400 \mathrm{nM}$ of aptamer-shRNA chimeras. After 48 hours, cells were either collected for qRT-PCR or seeded in 96-well plates at 2,000 cells/ well. 24 hours later, cells were irradiated with 6 Gy using a Gammacell 40 (Nordion) $137 \mathrm{Cs}$ radiator at approximately $0.6 \mathrm{~Gy} / \mathrm{min}$. Cell viability was assessed after 12 days by MTS.

Dicer processing analysis. For in vitro Dicer assay, $1 \mu \mathrm{g}$ of each aptamer-shRNA chimera was incubated with recombinant human Dicer following the manufacturer's recommendations (Recombinant Human Turbo Dicer Kit; GTS). For cellular Dicer assay, RNA from aptamer-shRNA chimera-treated LNCaP cells (as described above) were evaluated by Northern blot. Probes were as follows: DNAPK siRNA antisense, 5'-TTCGGCTAACTCGCCAGTTTA-3'; control siRNA antisense, 5'-AATTCTCCGAACGTGTCACGT-3'.

$5^{\prime}$ RACE. mRNA $(5 \mu \mathrm{g})$ from LNCaP cells or LNCaP tumor treated with aptamer-shRNA chimeras was ligated to GeneRacer adaptor (Invitrogen). Ligated RNA was reverse transcribed using a gene-specific primer (GSP[DNAPK] reverse 1, 5'-GGAGGGCTCCTTGACAAACACATCCAT-3'). To detect cleavage products, PCR was performed using primers complementary to the RNA adaptor (GR 5' primer, 5'-CTCTAGAGCGACTGGAGCACGAGGACACTA-3') and gene-specific primer (GSP[DNAPK] reverse 2, 5'-GGAAGGCCCGGAGTGCGTGTACCAT-3'). Amplification products were resolved by agarose gel electrophoresis, visualized by ethidium bromide staining, and confirmed by sequencing.

Animal model studies. Studies were performed according to the protocols approved by the Animal Care and Use Committee at Johns Hopkins University. 8-week-old athymic nude mice ( $(n u / n u$; Harlan Laboratories Inc.) were obtained from the Animal Center Isolation Facility at Johns Hopkins University and maintained in a sterile environment according to guidelines established by the Association for Assessment and Accreditation of Laboratory Animal Care. Mice were inoculated with $5 \times 10^{6}(50 \%$ Matrigel) PC3 cells or LNCaP cells subcutaneously, and tumors were grown to at least $0.8 \mathrm{~cm}$ in diameter. For aptamer-shRNA chimera knockdown, tumors were injected with 200 pmol chimeras on days -3 and -2 . On day 0 , the tumor was harvested and partitioned for RNA extraction or formalin fixation. For radiosensitization, LNCaP or PC3 tumors were randomized into no-radiation and radiation groups and treated with aptamer-shRNA chimeras as above. On day 0 , radiation groups received 6 Gy local IR $(5.8 \mathrm{~Gy} / \mathrm{min})$ to the tumor-bearing leg from a J.L. Shepherd Mark 137Cs irradiator with the remainder of the body shielded from the source. Tumors were measured every 2 days to calculate tumor volume: $(w \times l \times b) \times 0.52$. Tumor response was determined as reaching 4 times its volume at the start of radiation treatment.

Immunohistochemistry. Paraffin-embedded sections $(4 \mu \mathrm{m})$ were taken from xenograft tumors or human tissues. Slides were deparaffinized and rehydrated through a series of ethanol gradients, then treated with $0.1 \%$ Tween 20 detergent in deionized water and incubated in Target Retrieval solution (Dako) and in steam (Black and Decker Vegetable Steamer), then washed in PBS with Tween. After $3 \%$ hydrogen peroxide incubation, primary antibody anti-DNAPK (Ab-2, mouse mAb; Calbiochem) was added to each slide. A second antibody, Powervision (Poly-HRP anti-mouse IgG; Leica Biosystems) was applied to the specimens according to the manufacturer's standard protocol. The staining was developed with diaminobenzidine (DAB kit; Vector Laboratories) and counterstained with Mayer hematoxylin. Images were captured for presentation using a Nikon 50i microscopy with Nikon NIS-Elements software and an attached charge-coupled device digital camera. Brightfield setting was the same for all images. For quantification of DNAPK, whole DAB staining slides were scanned via ScanScope CS system (Aperio Technologies Inc.) at the Tissue Micro Array Core of Johns Hopkins University School of Medicine, and total DNAPK expression per cell nucleus was measured from 5-8 areas of tissue specimen for 500-1,000 cells using Framework for Image Dataset Analysis (FrIDA) software (http://bui2. win.ad.jhu.edu/frida/) as previously described (29). 
Ex vivo human prostate tissue model. Fresh human prostate tissue samples were obtained from the Department of Pathology of Johns Hopkins University. This study was approved by the Institutional Review Board at Johns Hopkins Medical Institution (approval no. NA_00015481), and informed consent was obtained from patients participating in the study. Fresh tissue representing histologically normal areas was bored from radical prostatectomy specimens and sliced at $300 \mu \mathrm{m}$ with a Krumdieck precision tissue slicer (Alabama Research and Development Corp.; ref. 30). The tissue slices were loaded onto titanium grids in 6-well plates containing culture medium with $200 \mathrm{nM}$ aptamer-shRNA chimeras and rotated on an inclined plane in a humidified tissue culture incubator at $37^{\circ} \mathrm{C}$ for 48 hours before being processed for immunohistochemical staining and quantification as above.

Statistics. Statistical analysis data of tumor size was evaluated by 2-way ANOVA. A $P$ value of 0.05 or less was considered significant. For the extension of tumor quadrupling experiments, events (animals whose tumor volume was not yet 4 -fold the size at injection) were plotted on a
Kaplan-Meier curve and analyzed by log-rank (Mantel-Cox) test. Paired samples were evaluated by 2 -tailed Student's $t$ test.

\section{Acknowledgments}

We thank Bruce Trock for discussion on library analysis; Neil Bander for providing PSMA antibody; Alan Meeker, Angelo De Marzo, Bora Gurel, and the IHC Core for assistance with analysis; and Ron Rodriguez for general discussions. This work was supported by NIH grant 5P50CA058236-15 and the David H. Koch Charitable Foundation.

Received for publication September 14, 2010, and accepted in revised form March 30, 2011.

Address correspondence to: Shawn E. Lupold, Johns Hopkins University School of Medicine, 600 N. Wolfe St., Park 209, Baltimore, Maryland 21287, USA. Phone: 410.502.4822; Fax: 410.502.7711; E-mail: slupold@jhmi.edu.
1. Ward JF, Moul JW. Rising prostate-specific antigen after primary prostate cancer therapy. Nat Clin Pract Urol. 2005;2(4):174-182.

2. Bolla $\mathrm{M}$, et al. Long-term results with immediate androgen suppression and external irradiation in patients with locally advanced prostate cancer (an EORTC study): a phase III randomised trial. Lancet. 2002;360(9327):103-106.

3. Walz J, et al. A nomogram predicting 10 -year life expectancy in candidates for radical prostatectomy or radiotherapy for prostate cancer.J Clin Oncol. 2007; 25(24):3576-3581.

4. Smith GC, Jackson SP. The DNA-dependent protein kinase. Genes Dev. 1999;13(8):916-934.

5. Helleday T, Lo J, van Gent DC, Engelward BP. DNA double-strand break repair: from mechanistic understanding to cancer treatment. DNA Repair (Amst). 2007;6(7):923-935.

6. Pollard JM, Gatti RA. Clinical radiation sensitivity with DNA repair disorders: an overview. Int J Radiat Oncol Biol Phys. 2009;74(5):1323-1331.

7. Chalmers AJ, Bentzen SM, Buffa FM. A general framework for quantifying the effects of DNA repair inhibitors on radiation sensitivity as a function of dose. Theor Biol Med Model. 2007;4:25.

8. Collis SJ, Swartz MJ, Nelson WG, DeWeese TL. Enhanced radiation and chemotherapy-mediated cell killing of human cancer cells by small inhibitory RNA silencing of DNA repair factors. Cancer Res. 2003;63(7):1550-1554

9. Ohnishi K, Scuric Z, Schiestl RH, Okamoto N, Takahashi A, Ohnishi T. siRNA targeting NBS1 or XIAP increases radiation sensitivity of human cancer cells independent of TP53 status. Radiat Res. 2006;166(3):454-462.

10. Dausse E, Da Rocha Gomes S, Toulme JJ. Aptamers: a new class of oligonucleotides in the drug discovery pipeline? Curr Opin Pharmacol. 2009;9(5):602-607.

11. Grimm D, Kay MA. Therapeutic application of RNAi: is mRNA targeting finally ready for prime time? J Clin Invest. 2007;117(12):3633-3641.

12. Zhou J, Rossi JJ. Aptamer-targeted cell-specific RNA interference. Silence. 2010;1(1):4

13. Lupold SE, Hicke BJ, Lin Y, Coffey DS. Identification and characterization of nuclease-stabilized RNA molecules that bind human prostate cancer cells via the prostate-specific membrane antigen. Cancer Res. 2002;62(14):4029-4033.

14. Cheng J, et al. Formulation of functionalized PLGA-PEG nanoparticles for in vivo targeted drug delivery. Biomaterials. 2007;28(5):869-876.
15. Chu TC, et al. Aptamer:toxin conjugates that specifically target prostate tumor cells. Cancer Res. 2006;66(12):5989-5992.

16. Chu TC, et al. Labeling tumor cells with fluorescent nanocrystal-aptamer bioconjugates. Biosens Bioelectron. 2006;21(10):1859-1866.

17. Farokhzad OC, et al. Targeted nanoparticleaptamer bioconjugates for cancer chemotherapy in vivo. Proc Natl Acad Sci U S A. 2006; 103(16):6315-6320.

18. Farokhzad OC, Jon S, Khademhosseini A, Tran TN, Lavan DA, Langer R. Nanoparticle-aptamer bioconjugates: a new approach for targeting prostate cancer cells. Cancer Res. 2004;64(21):7668-7672.

19. Chu TC, Twu KY, Ellington AD, Levy M. Aptamer mediated siRNA delivery. Nucleic Acids Res. 2006; 34(10):e73.

20. Dassie JP, et al. Systemic administration of optimized aptamer-siRNA chimeras promotes regression of PSMA-expressing tumors. Nat Biotechnol. 2009;27(9):839-849.

21. McNamara JO 2nd, et al. Cell type-specific delivery of siRNAs with aptamer-siRNA chimeras. Nat Biotechnol. 2006;24(8):1005-1015.

22. Pastor F, Kolonias D, Giangrande PH, Gilboa E. Induction of tumour immunity by targeted inhibition of nonsense-mediated mRNA decay. Nature. 2010;465(7295):227-230.

23. Wullner U, Neef I, Eller A, Kleines M, Tur MK Barth S. Cell-specific induction of apoptosis by rationally designed bivalent aptamer-siRNA transcripts silencing eukaryotic elongation factor 2 . Curr Cancer Drug Targets. 2008;8(7):554-565.

24. Bostwick DG, Pacelli A, Blute M, Roche P, Murphy GP. Prostate specific membrane antigen expression in prostatic intraepithelial neoplasia and adenocarcinoma: a study of 184 cases. Cancer. 1998; 82(11):2256-2261.

25. Perner $S$, et al. Prostate-specific membrane antigen expression as a predictor of prostate cancer progression. Hum Pathol. 2007;38(5):696-701.

26. Mu D, Hsu DS, Sancar A. Reaction mechanism of human DNA repair excision nuclease. J Biol Chem. 1996;271(14):8285-8294

27. Sledz CA, Holko M, de Veer MJ, Silverman RH, Williams BR. Activation of the interferon system by short-interfering RNAs. Nat Cell Biol. 2003; 5(9):834-839.

28. Chang SS, Reuter VE, Heston WD, Bander NH, Grauer LS, Gaudin PB. Five different anti-prostatespecific membrane antigen (PSMA) antibodies con- firm PSMA expression in tumor-associated neovasculature. Cancer Res. 1999;59(13):3192-3198.

29. Gurel B, et al. Nuclear MYC protein overexpression is an early alteration in human prostate carcinogenesis. Mod Pathol. 2008;21(9):1156-1167.

30. Kiviharju-af Hallstrom TM, et al. Human prostate epithelium lacks Wee1A-mediated DNA damageinduced checkpoint enforcement. Proc Natl Acad Sci U S A. 2007;104(17):7211-7216

31. Leibel SA, Hanks GE, Kramer S. Patterns of care outcome studies: results of the national practice in adenocarcinoma of the prostate. Int J Radiat Oncol Biol Phys. 1984;10(3):401-409.

32. Smit WG, Helle PA, van Putten WL, Wijnmaalen AJ, Seldenrath JJ, van der Werf-Messing BH. Late radiation damage in prostate cancer patients treated by high dose external radiotherapy in relation to rectal dose. Int J Radiat Oncol Biol Phys. 1990; 18(1):23-29.

33. Hoeijmakers JH. Genome maintenance mechanisms for preventing cancer. Nature. 2001; 411(6835):366-374

34. van Gent DC, Hoeijmakers JH, Kanaar R. Chromosomal stability and the DNA double-stranded break connection. Nat Rev Genet. 2001;2(3):196-206.

35. Vilenchik MM, Knudson AG. Endogenous DNA double-strand breaks: production, fidelity of repair, and induction of cancer. Proc Natl Acad Sci U S A. 2003;100(22):12871-12876.

36. Aagaard L, Rossi JJ. RNAi therapeutics: principles, prospects and challenges. Adv Drug Deliv Rev. 2007; 59(2-3):75-86

37. Castanotto D, Rossi JJ. The promises and pitfalls of RNA-interference-based therapeutics. Nature. 2009; 457(7228):426-433

38. Jinek M, Doudna JA. A three-dimensional view of the molecular machinery of RNA interference. Nature. 2009;457(7228):405-412

39. Siomi H, Siomi MC. On the road to reading the RNA-interference code. Nature. 2009; 457(7228):396-404.

40. Behlke MA. Chemical modification of siRNAs for in vivo use. Oligonucleotides. 2008;18(4):305-319.

41. Reynolds A, Leake D, Boese Q, Scaringe S, Marshall WS, Khvorova A. Rational siRNA design for RNA interference. Nat Biotechnol. 2004;22(3):326-330.

42. Soundararajan S, Chen W, Spicer EK, CourtenayLuck N, Fernandes DJ. The nucleolin targeting aptamer AS1411 destabilizes Bcl-2 messenger RNA in human breast cancer cells. Cancer Res. 2008;68(7):2358-2365. 